
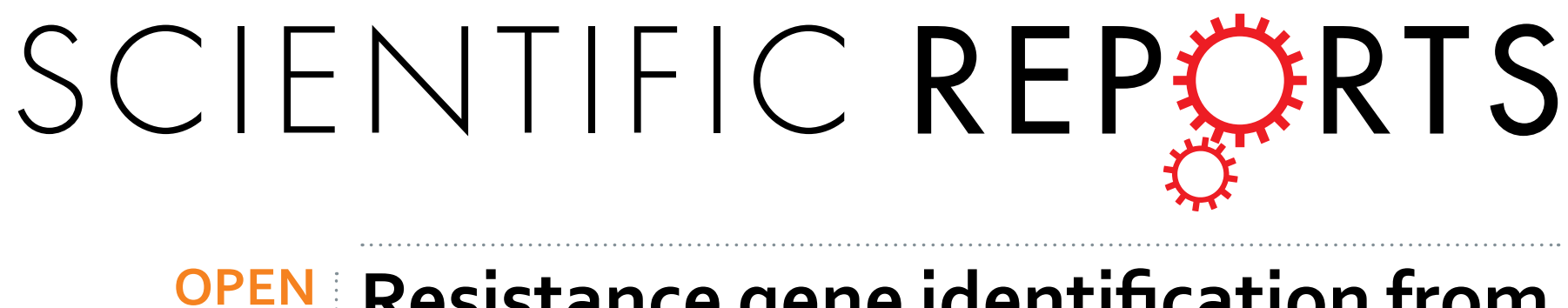

\title{
Resistance gene identification from Larimichthys crocea with machine learning techniques
}

Received: 01 June 2016

Accepted: 08 November 2016

Published: 06 December 2016
Yinyin $\mathrm{Cai}^{1,2, *}$, Zhijun Liao ${ }^{3, *}$, Ying Ju' ${ }^{1}$, Juan Liu ${ }^{4}$, Yong Mao ${ }^{2,5}$ \& Xiangrong Liu ${ }^{1,2}$

The research on resistance genes (R-gene) plays a vital role in bioinformatics as it has the capability of coping with adverse changes in the external environment, which can form the corresponding resistance protein by transcription and translation. It is meaningful to identify and predict R-gene of Larimichthys crocea (L.Crocea). It is friendly for breeding and the marine environment as well. Large amounts of L.Crocea's immune mechanisms have been explored by biological methods. However, much about them is still unclear. In order to break the limited understanding of the L.Crocea's immune mechanisms and to detect new R-gene and R-gene-like genes, this paper came up with a more useful combination prediction method, which is to extract and classify the feature of available genomic data by machine learning. The effectiveness of feature extraction and classification methods to identify potential novel R-gene was evaluated, and different statistical analyzes were utilized to explore the reliability of prediction method, which can help us further understand the immune mechanisms of L.Crocea against pathogens. In this paper, a webserver called LCRG-Pred is available at http://server.malab.cn/rg_Ic/.

Larimichthys crocea is a primary economic fish species in China ${ }^{1}$, belonging to vertebrates. However, with the expansion of breeding scale, in particular the abuse of antibiotics, parasite as well as viruses and bacteria ${ }^{1-3}$, pathogens have become a major constraint in the sustainable development of aquaculture of L.Crocea. Resistance genes play a key role in L.Crocea's immune system by transcribing to form resistance protein that contain Antimicrobial peptides (AP), Major histocompatibility complex (MHC), Immunoglobulin (Ig), Natural resistance associated macrophage protein (Nramp), Interferon (IFN), Lectin, Interleukins (ILs), tumour necrosis factors (TNFs), Lysozyme and etc. The expression of these genes can empower the organism against drugs or malnourished environment, such as antibiotics and communicable diseases, which are commonly used as selective genetic markers for developing excellent antibody strain. Despite advances in science, substantial genomic and transcriptome sequences call for genetic analyses in Larimichthys crocea ${ }^{4}$, and research on R-genes and R-gene-like genes can offer helpful understanding about the defense mechanisms of L.Crocea. These can not only meet breeding needs, but also the needs of life.

Certain methods have been utilized for R-gene mining, including experiment methods like protein/gene fusion $^{5,6}$, sequence assembly ${ }^{4,7}$, sequence alignment $/$ similarity $^{8,9}$, and structure-based approach ${ }^{10,11}$, etc. Because of biological mining methods are time-consuming and expensive for genome identification, machine learning methods are developed much more efficiently in classification and prediction of R-gene. The classifiers ${ }^{12}$, e.g. Support vector machine ${ }^{13-17}$, Naive bayes ${ }^{18,19}$ and Random forest ${ }^{20-22}$ were applied. Despite recent advances and applications mainly focus on plant resistance genes such as Xia et al. ${ }^{13}$ and Torres-Avilés et al. ${ }^{23}$ predicted R-gene in rice and tomato separately, and NBSPred ${ }^{24}$ was proposed to predict R-gene of plant. Lii et al. ${ }^{25}$ and Thorsten et al. ${ }^{26}$ suggest that there exist several emerging similarities in plant R-gene and animal innate immune receptor complexes. Robertsen ${ }^{27}$ found that the IFNs producing cells of fishes and IFNs gene structure were similar to those in mammals, and the deduced protein of fishes was highly homologous to mammalian. This means that a limited number of all known R-gens can be a likely explanation for identifying the immune system of L.Crocea.

\footnotetext{
${ }^{1}$ School of Information Science and Technology, Xiamen University, Xiamen, Fujian 361005, China. ${ }^{2}$ State Key Laboratory of Large Yellow Croaker Breeding, Ningde Fufa Fisheries Company Limited, Ningde, 352000, China. ${ }^{3}$ Department of Biochemistry and Molecular Biology, School of Basic Medical Sciences, Fujian Medical University, Fuzhou, 350122, China. ${ }^{4}$ School of Aerospace Engineering, Xiamen University, Xiamen, Fujian 361005, China. ${ }^{5}$ College of Ocean and Earth Sciences, Xiamen University, Xiamen, 361102, China. ${ }^{*}$ These authors contributed equally to this work. Correspondence and requests for materials should be addressed to Y.M. (email: maoyong@ xmu.edu.cn) orX.L. (email:xrliu@xmu.edu.cn)
} 


\section{Data-preprocessing}
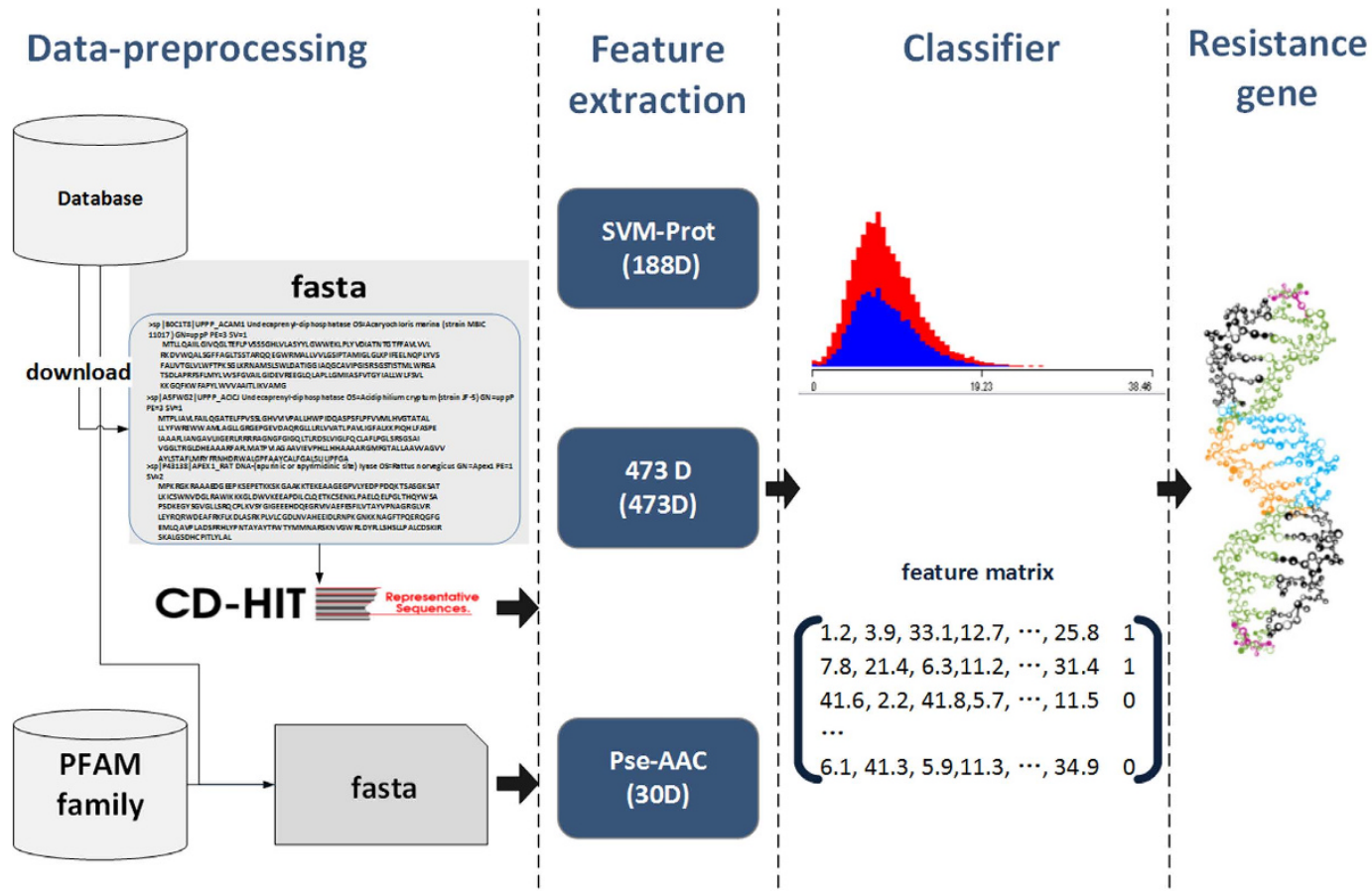

Figure 1. The main flowchart of the identification process.

\begin{tabular}{|l|c|c|c|c|c|c|}
\hline \multirow{2}{*}{ Sampling Method } & \multicolumn{2}{|c|}{ Training set } & \multicolumn{4}{c|}{ Accuracy } \\
\cline { 2 - 7 } & Resistance gene & Non-Resistance gene & SN & SP & Accuracy (\%) & ROC Area \\
\hline Original instance & 6720 & 10028 & 0.821 & 0.696 & 77.0898 & 0.855 \\
\hline Random-under-sampling & 6720 & 6720 & 0.831 & 0.687 & 75.878 & 0.850 \\
\hline Weighted random-sampling & 6720 & 10028 & 0.767 & 0.761 & 76.3974 & 0.854 \\
\hline
\end{tabular}

Table 1. Results based on three different sampling methods using random forest.

Considering these and other similarities, as a solution, machine leaning was used to model all reviewed resistance genes in all species, and the model was evaluated and applied to identify L.Crocea for novel R-gene.

This paper aims to identify and analyze the resistance genes of Larimichthys crocea so as to improve its own immune system to fight against the invasion of pathogens. In view of the specific functional classes of proteins with common structure and physical-chemical characteristics, we extract feature information from all known R-gene sequences with machine learning methods, and classification algorithms are adopted for identification of the gene fragment separately. Potential rules of the sequences could be acquired by studying the reviewed sequences, and the same properties were able to confirm by using the classifier model we obtained to classify the unknown sequence. Moreover, different feature extraction methods and classification methods were compared, and the results and differences of the prediction are discussed and analyzed. In addition, the quality of the prediction was verified. The main flowchart of the process is given in Fig. 1. In short, experiments demonstrate that the proposed methods, especially the SVMProt-RF by using SVM-Prot ${ }^{28,29}$ combined with Random forest, could be utilized for the prediction of novel R-gene.

\section{Results}

Comparative Analysis. Sampling method Comparative Analysis. Firstly, on the basis of SVM-Prot feature method, we compared the performance of original samples $\left(\Omega_{0 \text { riglR-g }}\right)$ and samples after two sampling strategies $\left(\Omega_{t r}\right.$ and $\left.\Omega_{w t r}\right)$ separately under Random forest classifier, where all other parameters are the same. Table 1 shows the results based on three different sampling methods. As we can see, given that the number of non-R-gene is greater than R-gene, it makes no sense if R-gene was classified as non-R-gene, though it gets higher accuracy. Besides, weighted random sampling contributes to the best result, which is good for establishment of a better performance classifier.

Multi-Classifier Comparative Analysis. In order to demonstrate the validity of the classification results of R-gene sequence in the Random forest algorithm, we compare the results of $\Omega_{\mathrm{tr}}$ treated by SVM-Prot feature under different classifiers. To get the objective evaluation, we adopt both test set $\Omega_{\text {test }}$ and 10 -fold cross-validation to verify the classification effect, as shown in Table 2 and Fig. 2. Visibly, the results of Random forest, LibD3C ${ }^{30}$, Bagging, Gradient Boosting Decision Tree (GBDT) and RandomSubSpace algorithm we obtained are better than others, 


\begin{tabular}{|l|c|c|c|c|c|c|}
\hline Classifier & Attributes & SN & SP & Mcc & Accuracy (\%) & ROC Area \\
\hline Random forest & 13440 & 0.831 & 0.687 & 0.523 & 75.878 & 0.850 \\
\hline LibD3C & 13440 & 0.820 & 0.700 & 0.524 & 76.0045 & 0.846 \\
\hline J48 & 13440 & 0.688 & 0.683 & 0.371 & 68.5491 & 0.678 \\
\hline Bayes Network & 13440 & 0.810 & 0.597 & 0.417 & 70.3646 & 0.761 \\
\hline Naive Bayes & 13440 & 0.882 & 0.264 & 0.185 & 57.2768 & 0.690 \\
\hline KNN-IB1 & 13440 & 0.639 & 0.765 & 0.408 & 70.2158 & 0.706 \\
\hline AdaBoostM1 & 13440 & 0.782 & 0.605 & 0.393 & 69.3601 & 0.763 \\
\hline Bagging & 13440 & 0.786 & 0.696 & 0.483 & 74.0699 & 0.822 \\
\hline GBDT & 13440 & 0.718 & 0.705 & 0.456 & 72.7902 & 0.818 \\
\hline Random tree & 13440 & 0.673 & 0.672 & 0.346 & 67.2842 & 0.673 \\
\hline RandomSubSpace & 13440 & 0.819 & 0.662 & 0.486 & 74.0179 & 0.826 \\
\hline SMO & 13440 & 0.677 & 0.749 & 0.427 & 71.2798 & 0.713 \\
\hline LibSVM & 13440 & 0.947 & 0.307 & 0.331 & 62.7232 & 0.627 \\
\hline
\end{tabular}

Table 2. Performance comparison of different classifier.

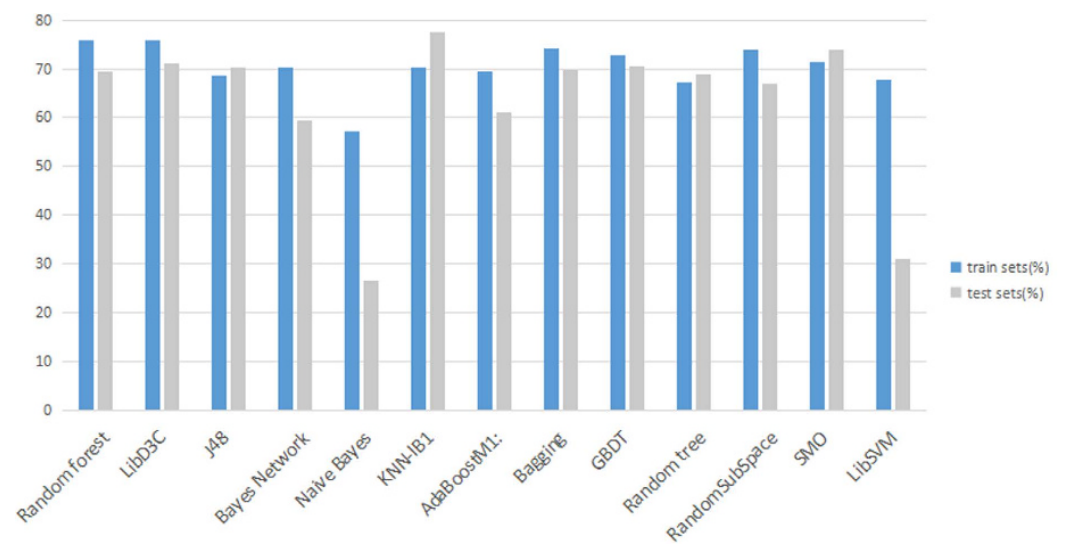

Figure 2. Performance of test sets on different classifiers.

their accuracies being $75.88 \%, 76.00 \%, 74.07 \%, 72.79 \%$ and $74.02 \%$ respectively, as shown in Table 2 . In view of the performance of classifier, the sensitivity of J48, KNN-IB1, Random tree, GBDT and SMO are all less than $72 \%$, that is, the model is less than $72 \%$ for classifying R-gene correctly, even if the total accuracy of some of these methods is very high. Besides, the sensitivities of Bayes Network, Naive Bayes, and LibSVM are higher than $80 \%$, but their low specificities result in a serious false positive problem when identifying the R-gene. Different from the above classifiers, Random forest, LibD3C, AdaboostM1, bagging and RandomSubSpace with the guarantee of high sensitivity have an acceptable specificity. In addition, Random forest and LibD3C work better considering the Mcc, total accuracy and ROC Area. Furthermore, for the time consumed, LibD3C is 36 times more than Random forest with the same parameters. For the test set, KNN-IB1 achieved a higher accuracy rate of 77.5998\% while Random forest 69.347\%, as can be seen in Fig. 2, which can only indicate that KNN-IB1 has a higher classification accuracy of non-R-gene. Therefore, the function of Random forest classifier shows better with comprehensive consideration.

Multi-Feature Comparative Analysis. In this section, feature extraction methods are compared in our experiment on the basis of Random forest classifier, including the 188-D constructed from SVM-Port features, Pse-AAC ${ }^{31}$ features and 473-D features, as shown in Table 3. The strengths of the 188-D feature extraction algorithm is obvious, which obtains higher accuracy as well as higher sensitivity and specificity, better than the other two feature extraction algorithms. The second part of Table 3 denotes the accuracy of the training set and test set in 188-D features and Pse-AAC and 473-D feature method under the Random forest classifiers. And the accuracy of the test set of Pse-AAC reached 60.913\% while SVM-Port features reached $69.347 \%$, and 473-D features reached 55\% respectively. We can learn that SVM-Prot features combined with Random forest have the best result among these algorithms through synthetical consideration. Here we call it SVMProt-RF method.

Identification R-gene from Larimichthys crocea. To get a better understanding of Larimichthys crocea immune system for future breeding and disease prevention, an effective support and recognition of the resistance genes of L.Crocea is particularly crucial. In our experiments, a combined classification model was developed by identifying all reviewed R-gene, and it was applied to screen the R-gene of L.Crocea. As for the selection of the original data of prediction model, we used the protein sequence coded by R-gene based on the following 


\begin{tabular}{|c|c|c|c|c|c|c|c|}
\hline \multirow[b]{2}{*}{ Feature extraction method } & \multirow[b]{2}{*}{ Dimension } & \multicolumn{2}{|c|}{ Training set } & \multicolumn{4}{|c|}{ Accuracy } \\
\hline & & Resistance gene & Non-Resistance gene & SN & SP & Mcc & Accuracy (\%) \\
\hline $188-\mathrm{D}$ & 188 & 6720 & 6720 & 0.831 & 0.687 & 0.523 & 75.878 \\
\hline Pse-AAC & 30 & 6720 & 6720 & 0.761 & 0.627 & 0.392 & 69.4345 \\
\hline \multirow{2}{*}{ 473-D } & 473 & 178 & 226 & 0.371 & 0.752 & 0.133 & 58.4158 \\
\hline & & \multicolumn{4}{|c|}{ test set } & \multirow{2}{*}{\multicolumn{2}{|c|}{ Accuracy (\%) }} \\
\hline Feature extraction method & Dimension & \multicolumn{2}{|c|}{ Resistance gene } & \multicolumn{2}{|c|}{ Non-Resistance gene } & & \\
\hline $188-\mathrm{D}$ & 188 & \multicolumn{2}{|c|}{0} & \multicolumn{2}{|c|}{3308} & \multicolumn{2}{|c|}{69.347} \\
\hline Pse-AAC & 30 & \multicolumn{2}{|r|}{0} & \multicolumn{2}{|c|}{3308} & \\
\hline $473-\mathrm{D}$ & 473 & \multicolumn{2}{|r|}{20} & \multicolumn{2}{|c|}{20} & \multicolumn{2}{|r|}{$\begin{array}{c}60.9129 \\
550\end{array}$} \\
\hline
\end{tabular}

Table 3. Performance comparison of 188-D features and 473-D features.

\begin{tabular}{|l|c|c|c|}
\hline \multirow{2}{*}{ Prediction model } & \multicolumn{3}{|c|}{ Accuracy } \\
\cline { 2 - 4 } & TP Rate & TN Rate & Accuracy (\%) \\
\hline$\Omega_{\text {OriglR-g }}$ model & 0.453 & 0.547 & 45.3047 \\
\hline$\Omega_{\mathrm{tr}}$ model & 0.646 & 0.354 & 64.6409 \\
\hline$\Omega_{\mathrm{wtr}}$ model & 0.546 & 0.454 & 54.3956 \\
\hline
\end{tabular}

Table 4. Prediction results of $\Omega_{\mathrm{LC}}$ under different data balancing models.

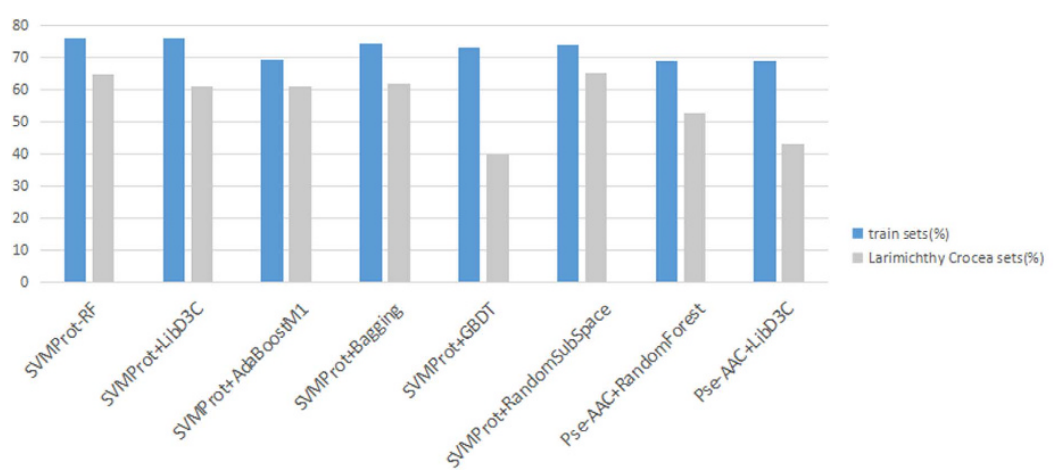

Figure 3. Prediction results of L.Crocea on different classification models.

conditions: R-gene expresses the resistance function through the protein product directly; protein sequence consists of 20 amino acid with abundant physicochemical properties, while nucleotide sequence consists of only 4 elements, which is not conducive to the feature extraction. Here, we obtained multiple hybrid prediction models with higher accuracy after a series of comparison as demonstrated before. $\Omega_{L C}$ (sequence of L.Crocea) was predicted based on these models. A comparison was made between SVMProt-RF method and others as well. Figure 3 gives the results of the prediction. As we can see, $64.64 \%$ R-gene existent in the sequences of L.Crocea while $61.01 \%, 61.12 \%, 61.68 \%, 39.74 \%, 65.16 \%, 52.70 \%$ and $43.20 \%$ were respectively obtained in others. Furthermore, Table 4 shows the prediction results of $\Omega_{L C}$ applied by $\Omega_{\text {origlR-g }}$ model, $\Omega_{t r}$ and $\Omega_{w t r}$ model, their prediction results taking up $45.30 \%, 64.64 \%$ and $54.39 \%$ respectively.

A comparative table of SVMProt-RF and NBSpred prediction is given in the Table 5, since there exist obvious similarities of pathogen-associated molecular patterns (PAMPs) in animals and plants, especially the plant receptors resembling mammalian Toll-like receptors (TLR) or cytoplasmic nucleotide-binding oligomerization domain leucine-rich repeat (LRR) proteins ${ }^{26}$, and NBSpred is a web server for predicting nucleotide binding site lucine-rich repeat proteins (NBS-LRR) of plant ${ }^{24}$. SVM method is used to extract features of datasets by calculating six compositional attributes, including amino acid frequency, dipeptide frequencies, tripeptide frequencies, multiplet frequencies and hydrophobicity composition ${ }^{24}$. Total, 9801 sequences are identified as R-gene and R-gene-like genes through SVMProt-RF. NBSPred only detected 2.544\% sequences as R-gene from L.Crocea dataset. Distinct differences remain in plants and vertebrates, such as plants do not own specific immunity and cannot produce immunizations because they lack circulatory blood system like an animal. So, we can find that one prediction model can identify R-gene of plants accurately but fails to predict R-gene of L.Crocea.

\section{Discussion}

In this paper, after comparison among different feature extraction methods and classification algorithms, the SVM-Prot feature extract method and random forests classification algorithm were combined (SVMProt-RF) 


\begin{tabular}{|l|c|c|c|}
\hline Dataset & $\begin{array}{c}\text { Number of } \\
\text { sequences }\end{array}$ & $\begin{array}{c}\text { SVMProt-RF } \\
\text { prediction }\end{array}$ & NBSPred prediction \\
\hline L.Crocea Dataset & 18018 & 9801 & $\begin{array}{c}457 \text { 17964 (total number } \\
\text { after NBSPred) }\end{array}$ \\
\hline Accuracy (\%) & & 54.3956 & 2.5440 \\
\hline
\end{tabular}

Table 5. Comparison of SVMProt-RM and NBSPred prediction for R-gene of L.Crocea.

to preliminarily mine the resistance gene of the whole protein data, which proves to achieve the best results. And further screening was conducted on the acquired resistance gene to determine the relationship between the candidate sequence and the resistance trait. The work was divided into the following parts: the establishment of resistance data sets, the feature extraction, the sampling of imbalanced data sets and the comparison of resistance genes classification models. In comparison with other previously mentioned works and methods, we can reach the conclusion that our methods have the following advantages:

(1) It reduce the redundancy of R-gene samples, and optimize efficiency by keeping the original data information.

(2) Feature extraction based on datasets that contains resistance genes of all reviewed species and the prediction of R-gene of L.Crocea are more accurate.

(3) Compared with other classifiers, the result of SVMProt-RF method associated with weight random-sampling shows that the model has a better sensitivity and specificity, and better adaptability to identify R-gene.

(4) It Can be used to predict the resistance genes of more candidate sequences, and verify the correlation between them with biological experiment.

The establishment of the model is of great significance for the subsequent resistance gene discovery and its evolution, regulation and pathway analysis. What's more, for the immune system-related genes of Larimichthys crocea, further exploration is still required.

\section{Method}

Data preprocessing. The original R-gene sequences were retrieved from Uniprot database ${ }^{32}$, which has been reviewed by experimentation. The dataset is composed of 13,959 sequences that contains all species like zoon, plants and fungi, denoted as $\Omega_{\text {riglR-g }}$. Each R-gene class, nevertheless, contains a lot of duplicate sequences that cause excessive redundancy. Therefore, CD-HIT was utilized to remove redundancy in positive dataset, which has been used in the realm of bioinformatics ${ }^{33,34}$. Considering the following algorithm: First, sort out all sequences according to their length; then form the classes by sequentially processing the length sequence. If the similarity of new sequence was higher than the existing class in threshold, the new sequence was added to this class, otherwise make it as a new class. Finally, 6720 R-gene were obtained with similarity below $70 \%$ after CD-HIT, denoted as $\Omega_{R-g}$ :

$$
\Omega_{R-g}=C D-H I T_{70 \%}\left(\Omega_{\text {OriglR-g }}\right)
$$

The negative sample was acquired from PFAM families due to the intimate relationship between R-gene and its protein sequence. No-duplicates PFAM of R-gene were removed from the whole PFAM families database. We got negative families here, and the longest sequence of proteins was fetched in each negative families. 10028 non-R-gene sequences were involved, denoted as $\Omega_{N R-g}$. Thus the training dataset $\Omega$ is denoted as follows:

$$
\Omega=\Omega_{R-g} \cup \Omega_{N R-g}
$$

where $\Omega$ contains a total of 16,748 sequences. The prediction datasets of Larimichthys crocea that consist of 18,018 sequences are collected from Uniprot database ${ }^{32}$ as well. To describe it simply, we denoted it as $\Omega_{L C}$.

Feature extraction algorithm. SVM-Prot features. SVM-Prot is a web server for protein classification. It constructs $188-\mathrm{D}$ features for protein sequences description and classification ${ }^{28,29}$. The features have been applied successfully in several protein identification works, such as cytokines ${ }^{35,36}$ and enzymes ${ }^{37,38}$. The extracted features include hydrophobicity, normalized van der Waals volume, polarity, polarizability, charge, surface tension, secondary structure and solvent accessibility ${ }^{28}$. For each of these 8 types of physical-chemical properties, some feature groups were designed to describe global information of protein sequences. These feature groups contain composition $(\mathrm{C})$, transition $(\mathrm{T})$ and distribution $(\mathrm{D})^{14,28}$. C expresses a percentage of the amino acids of particular property over total amino acid sequence. $\mathrm{T}$ is the frequency of amino acids of particular property that are intimately next to another amino acid of particular property. D depicts the position of amino acids of particular property in their sequences. Thus, the dimension of each feature vector is 21 (denoted as $D_{\text {eachV }}$ ). In addition, considering amino acid composition (denoted as $H_{\text {acc }}$ ), the protein structure is composed of 20 amino acids: $\mathrm{A}, \mathrm{C}$, D, E, F, G, H, I, K, L, M, N, P, Q, R, S, T, V, W, Y ${ }^{39,40}$. So the dimension of 188-D features is

$$
D_{188-D}=\sum_{\mathrm{i}=1}^{L} D_{e a c h V}+H_{a a c}
$$




\begin{tabular}{|l|c|c|c|c|c|c|c|}
\hline Property & \multicolumn{7}{|c|}{ Value of feature vector } \\
\hline \multirow{3}{*}{ amino acid composition } & 9.3664 & 0.2755 & 1.6529 & 3.5813 & 6.0606 & 8.5399 & 3.8567 \\
\cline { 2 - 8 } & 7.1625 & 0.8264 & 12.1212 & 4.6832 & 3.5813 & 4.9587 & 2.7548 \\
\cline { 2 - 8 } & 3.3058 & 9.3664 & 6.8871 & 5.5096 & 2.7548 & 2.7548 & \\
\hline \multirow{3}{*}{ Hydrophobic } & 15.7025 & 45.7300 & 38.5675 & 12.9834 & 12.4309 & 37.5690 & 1.6529 \\
\cline { 2 - 8 } & 29.2011 & 62.8099 & 82.6446 & 97.5207 & 0.5510 & 24.7934 & 49.5868 \\
\cline { 2 - 8 } & 73.0027 & 100.0 & 1.6529 & 25.3443 & 52.066 & 75.7576 & 99.1735 \\
\hline \multirow{3}{*}{ Van der Waals volume } & 0.2755 & 28.9256 & 50.9642 & 74.1047 & 99.4490 & 41.3223 & 39.1185 \\
\cline { 2 - 8 } & 19.5592 & 33.9779 & 17.6796 & 12.7072 & 0.2755 & 23.4160 & 45.1791 \\
\cline { 2 - 8 } & 72.1763 & 99.4490 & 0.5510 & 23.1405 & 48.4848 & 73.8292 & 100.0 \\
\hline
\end{tabular}

Table 6. Feature of PSBA1 R-gene in Acaryochloris marina.

where $L$ is the number of features. The features of $\Omega$ and $\Omega_{L C}$ were extracted. Table 6 shows a part of the results of PSBA1 R-gene in Acaryochloris marina.

Pseudo amino acid composition features. Pseudo amino acid composition features (Pse-AAC) ${ }^{41}$ as an efficient computation tool has been diffusely leveraged for protein sequences in predicting protein structures and functions $^{31,41}$, as well as DNA and RNA sequences ${ }^{42}$. To describe it distinctly, we assume a R-gene sequence $R$, expressed as:

$$
R=r_{1} r_{2} r_{3} r_{4} r_{5} \ldots r_{L}
$$

here, $L$ denotes the length of the sequence and $r_{i}(i=1,2, \ldots, L$ is the position of residue in $R$. Besides, given the different amphiphilic features of proteins, the Pse-AAC feature of $R$ can be defined as the following vector ${ }^{41,42}$ :

$$
\begin{gathered}
\text { feature }=\left[F_{1} \ldots F_{20} F_{20+1} \ldots F_{20+\lambda} F_{20+\lambda+1} \ldots F_{20+2 \lambda}\right]^{T} \\
\mathrm{~F}_{\xi}=\frac{\Gamma_{\xi}}{\sum_{i=1}^{20} \mathrm{f}_{i}+\omega \sum_{j=1}^{2 \lambda} e_{j}}, \xi=1,2, \ldots, 20+2 \lambda \\
\Gamma_{\xi}=\left\{\begin{array}{l}
f_{\xi}, 1 \leq \xi \leq 20 \\
\omega e_{\xi-20}, 20+1 \leq \xi \leq 20+2 \lambda
\end{array}\right.
\end{gathered}
$$

where $f_{i}(1 \leq i \leq 20)$ denotes the frequency of the 20 amino acids in $R$, and $\lambda$ is the top counted rank of the correlational protein sequences. We have a 30 dimension feature vector in this experiment. $\omega$ represents the weight factor, and $e_{j}$ depicts the correlation factor among residues of protein sequences. Features of R-gene were extracted by this feature representation method, which sufficiently incorporates the effects of sequence order.

Data Balancing. The unbalanced data problem always has huge impact on the result of the classification ${ }^{43}$. The classifiers tend to have a higher recognition rate for the majority class, which make it hard to identify the minority class correctly ${ }^{44,45}$. What we want is to eliminate the over fitting problem caused by unbalanced data. The commonly used method is sampling ${ }^{46}$, including under-sampling and over-sampling.

Since it is easy to obtain reviewed R-gene but not the non-R-gene, which incurs serious class imbalance problem and affects the performance of the classifier, two sampling methods are used in this paper to find out the best performance. One is random-under-sampling. The balance of the train sets is realized by random sampling of large class set, where the number of large class sets equals the small class sets. Here we get 6720 sequences each for $\Omega_{N R-g}$ and $\Omega_{R-g}$ as train sets, denoted as $\Omega_{\mathrm{tr}}$ and 3308 negative sequences remain as test sets $\Omega_{\text {test }}$. Another method we applied is weighted random sampling ${ }^{47}$, balancing the dataset by adding different weights to the unbalanced samples. Seeing that the ratio about $\Omega_{R-g}$ and $\Omega_{N R-g}$ is approximately equal to 7:10, weight factor 10 and 7 were added to the $\Omega_{R-g}$ and $\Omega_{N R-g}$ separately, so 16748 train sets were obtained, denoted as $\Omega_{\mathrm{wtr}}$.

Classifier selection and tools. Random forest. Random forest is a kind of classifier which is trained and predicted by a number of trees, as proposed by Leo Breiman ${ }^{48}$. Numerous advantages have been listed than other algorithms, including noise-ability, avoiding over-fitting, being able to handle high dimensional (feature) data and etc. The essence in this algorithm is an improvement based on the decision tree. An object can be categorized into a class, when the class follows the principle of the judgment based on every decision tree in the forest. The classification ability of the single tree would be marginal, but the probability of being classified properly is greatly enhanced after random generation of a large number of decision trees. In this study, R-gene is a binary classification, so all decision trees are binary tree.

WEKA. WEKA is one of the well-known data mining platform (http://www.cs.waikato.ac.nz/ml/weka/) that are utilized for data analysis and model prediction. Several machine learning algorithms were gathered as tools. 


\begin{tabular}{|l|c|c|}
\hline Classification & $\begin{array}{c}\text { Positive instance of } \\
\text { prediction }\end{array}$ & $\begin{array}{c}\text { Negative instance of } \\
\text { prediction }\end{array}$ \\
\hline Positive instance & $T P_{i}$ & $F N_{i}$ \\
\hline Negative instance & $F P_{i}$ & $T N_{i}$ \\
\hline
\end{tabular}

Table 7. Confusion matrix of binary classification performance of R-gene.

Cross-validation is provided by WEKA. In this study, we utilize its classification function to establish a model of $\Omega_{\mathrm{tr}}$ and its test sets $\Omega_{\text {test }}$ to verify the precision of the model. Thirteen classifiers are selected for this paper.

Measurement. Sensitivity (SN), specificity (SP), overall accuracy (Acc) and Matthew's correlation coefficient (Mcc) are usually applied in bioinformatics ${ }^{49-55}$ to measure the function of the classifier. Given datasets $S=s_{1}, s_{2}, s_{3}, s_{4}, \ldots, s_{m}, m$ is the number of samples. Based on the confusion matrix of binary classification performance of R-gene (shown in Table 7), we have:

$$
\begin{array}{ll}
T P_{i}=\sum_{i=1}^{m} s_{i i} & F P_{i}=\sum_{i=1}^{m} s_{i j} \\
F N_{i}=\sum_{i=1}^{m} s_{j i} & T N_{i}=\sum_{i=1}^{m} s_{j j}
\end{array}
$$

where $T P_{i}, F P_{i}, T N_{i}, F N_{i}$ denote the numbers of true positive instances, false positive instances, true negative instances and false negative instances respectively. The first subscript of $s_{i i}$ indicates the prediction result and the second indicates the true class of sample $s_{m}$. And we have ${ }^{14,56}$ :

$$
\begin{gathered}
S N=\frac{T P_{i}}{T P_{i}+F P_{i}} \\
S P=\frac{T N_{i}}{F P_{i}+T N_{i}} \\
A c c=\frac{T P_{i}+T N_{i}}{T P_{i}+F P_{i}+T N_{i}+F N_{i}} \\
M c c=\frac{T P_{i} \times T N_{i}-F P_{i} \times F N_{i}}{\sqrt{\left(T P_{i}+F P_{i}\right) \times\left(T N_{i}+F N_{i}\right) \times\left(T P_{i}+F N_{i}\right) \times\left(T N_{i}+F P_{i}\right)}}
\end{gathered}
$$

\section{References}

1. J. F. Liu \& K. H. Han. Current development situation and countermeasure of large yellow crocker industry in China. Journal of Fujian Fisheries 33, 1006 -5601 (2011).

2. X. Dong et al. Anti-infective mannose receptor immune mechanism in large yellow croaker (Larimichthys crocea). Fish \& Shellfish Immunology 54, 257-265 (2011).

3. Deng et al. Bacterial composition in large yellow croaker (Larimichthys crocea) culture water. Journal of Fishery Sciences of China 21, 1277-1288 (2014).

4. Z. Han et al. De novo characterization of Larimichthys crocea transcriptome for growth-/immune-related gene identification and massive microsatellite (SSR) marker development. Chinese Journal of Oceanology and slimnology 1-10 (2016).

5. A. J. Enright, I. Iliopoulos, N. C. Kyrpides \& C. A. Ouzounis. Protein interaction maps for complete genomes based on gene fusion events. Nature 402, 86-90 (1999).

6. M. Veena, P. Melvin, S. Shailasree, K. Ramach \& r. Kini. Cloning, expression and purification of resistance gene analogue RGPM 301 from pearl millet in Escherichia coli. J App Biol Biotech 4, 053-059 (2016).

7. C. Wu et al. The draft genome of the large yellow croaker reveals well-developed innate immunity. Nature Communications $\mathbf{5}$, 5227-5227 (2014).

8. A. D. Baxevanis \& B. Ouellette. Practical aspects of multiple sequence alignment. Methods of Biochemical Analysis 39, 172-188 (1998).

9. D. L. Zhang, C. H. Lv, D. h. Yu \& Z. Y. Wang. Characterization and functional analysis of a tandem-repeat galectin-9 in large yellow croaker Larimichthys crocea. Fish and Shellfish Immunology 52, 167-178 (2016).

10. M. C. Franklin et al. Structural Genomics for Drug Design against the Pathogen Coxiella burnetii. Proteins-structure Function \& Bioinformatics 83, 2124-2136 (2015).

11. S. I. Elshahawi et al. Structure-guided functional characterization of enediyne self-sacrifice resistance proteins, CalU16 and CalU19. Acs Chemical Biology 9, 2347-2358 (2014).

12. X. Wen, L. Shao, Y. Xue \& W. Fang. A rapid learning algorithm for vehicle classification. Information Sciences 295, 395-406 (2015).

13. J. Xia, X. Hu, F. Shi, X. Niu \& C. Zhang. Support vector machine method on predicting resistance gene against Xanthomonas oryzae pv. oryzae in rice. Expert Systems with Applications 37, 5946-5950 (2010).

14. H. H. Lin, L. Y. Han, C. Z. Cai, Z. L. Ji \& Y. Z. Chen. Prediction of transporter family from protein sequence by support vector machine approach. Proteins 62, 218-31 (2006).

15. W. Chen, P. M. Feng, E. Z. Deng, H. Lin \& K. C. Chou. iTIS-PseTNC: a sequence-based predictor for identifying translation initiation site in human genes using pseudo trinucleotide composition. Analytical Biochemistry 462, 76-83 (2014).

16. B. Gu et al. Incremental learning for $v$-Support Vector Regression. Neural Networks the Official Journal of the International Neural Network Society 67, 140-150 (2015). 
17. B. Gu, V. S. Sheng, K. Y. Tay, W. Romano \& S. Li. Incremental Support Vector Learning for Ordinal Regression. IEEE Transactions on Neural Networks \& Learning Systems 26, 1403-1416 (2014).

18. C. D. Nguyen, K. J. Gardiner, D. Nguyen \& K. J. Cios. Prediction of Protein Functions from Protein Interaction Networks: A Naive Bayes Approach. Lecture Notes in Computer Science 5351, 788-798 (2008).

19. H. Geng, T. Lu, X. Lin, Y. Liu \& F. Yan. Prediction of Protein-Protein Interaction Sites Based on Naive Bayes Classifier. Biochemistry Research International 2015, 1-7 (2015).

20. Y. Qi. Random Forest for Bioinformatics. Ensemble Machine Learning: Methods and Applications 307-323 (2012).

21. Y. Guo, X. Liu \& M. Guo. Identification of Plant Resistance Gene with Random Forest. Journal of Frontiers of Computer Science \& Technology 6, 67-77 (2012).

22. J. Ahoi. Computational prediction of protein phosphorylation site using random forest. Dissertations \& Theses - Gradworks (2015).

23. F. Torres-Avilés, J. S. Romeo \& L. López-Kleine. Data mining and influential analysis of gene expression data for plant resistance gene identification in tomato (Solanum lycopersicum). Electronic Journal of Biotechnology 17, 79-82 (2014).

24. S. K. Kushwaha, P. Chauhan, K. Hedlund \& D. Ahrén. NBSPred: a support vector machine-based high-throughput pipeline for plant resistance protein NBSLRR prediction. Bioinformatics 32, 1223-1225 (2015).

25. B. F. Holt Iii, D. A. Hubert \& J. L. Dangl. Resistance gene signaling in plants - complex similarities to animal innate immunity. Current Opinion in Immunology 15, 20-25 (2003).

26. T. Nürnberger, F. Brunner, B. Kemmerling \& L. Piater. Innate immunity in plants and animals: striking similarities and obvious differences. Immunological Reviews 198, 249-66 (2004).

27. B. Robertsen. The interferon system of teleost fish. Fish \& Shellfish Immunology 20, 172-91 (2006).

28. C. Z. Cai, L. Y. Han, Z. L. Ji, X. Chen \& Y. Z. Chen. SVM-Prot: web-based support vector machine software for functional classification of a protein from its primary sequence. Nucleic Acids Research 31, 3692-3697 (2003).

29. Y. H. Li et al. SVM-Prot 2016: A Web-Server for Machine Learning Prediction of Protein Functional Families from Sequence Irrespective of Similarity. Plos One 11 (2016).

30. C. Lin et al. LibD3C: Ensemble classifiers with a clustering and dynamic selection strategy. Neurocomputing 123, 424-435 (2014).

31. D. Pufeng, G. Shuwang \& J. Yasen. PseAAC-General: Fast Building Various Modes of General Form of Chou's Pseudo-Amino Acid Composition for Large-Scale Protein Datasets. International Journal of Molecular Sciences 15, 3495-506 (2014).

32. U. P. Consortium. The Universal Protein Resource (UniProt) 2009. Nucleic Acids Research 36, D154-D159 (2008)

33. W. Chen, P. Feng, H. Tang, H. Ding \& H. Lin. RAMPred: identifying the N(1)-methyladenosine sites in eukaryotic transcriptomes. Sci Rep 6, 31080 (2016)

34. W. Chen, H. Ding, P. Feng, H. Lin \& K. C. Chou. iACP: a sequence-based tool for identifying anticancer peptides. Oncotarget 7, 16895-909 (2016).

35. Q. Zou et al. An approach for identifying cytokines based on a novel ensemble classifier. BioMed research international 2013, 686090 (2013).

36. X. Zeng, S. Yuan, X. Huang \& Q. Zou. Identification of cytokine via an improved genetic algorithm. Frontiers of Computer Science 9 , 643-651 (2015).

37. X.-Y. Cheng et al. A global characterization and identification of multifunctional enzymes. PLoS One 7, e38979 (2012).

38. Q. Zou, W. Chen, Y. Huang, X. Liu \& Y. Jiang. Identifying Multi-functional Enzyme with Hierarchical Multi-label Classifier. Journal of Computational and Theoretical Nanoscience 10, 1038-1043 (2013).

39. Y. Huang et al. Biological functions of microRNAs: a review. Journal of Physiology and Biochemistry 67, 129-139 (2011).

40. A. K. Arakaki, Y. Huang \& J. Skolnick. EFICAz 2: enzyme function inference by a combined approach enhanced by machine learning. Bmc Bioinformatics 10, 1-15 (2009).

41. C. Kuo-Chen. Using amphiphilic pseudo amino acid composition to predict enzyme subfamily classes. Bioinformatics 21, 10-19 (2005).

42. L. Bin et al. Pse-in-One: a web server for generating various modes of pseudo components of DNA, RNA, and protein sequences. Nucleic Acids Research 43, 65-71 (2015).

43. L. Song et al. nDNA-prot: Identification of DNA-binding Proteins Based on Unbalanced Classification. BMC Bioinformatics 15, 298 (2014).

44. Q. Zou, M. Guo, Y. Liu \& Jun Wang. A Classification Method for Class-Imbalanced Data and Its Application on Bioinformatics. Journal of Computer Research \& Development 47, 1407-1414 (2010).

45. S. Lin et al. Under-sampling Method Research in Class-Imbalanced Data. Journal of Computer Research \& Development 47-53 (2011).

46. G. E. A. P. A. Batista, R. C. Prati \& M. C. Monard. A study of the behavior of several methods for balancing machine learning training data. Acm Sigkdd Explorations Newsletter 6, 20-29 (2004).

47. L. Guo, N. I. Ziwei, Y. Jiang \& Q. Zou. Research on Imbalanced Data Classification Based on Ensemble and Under-Sampling. Journal of Frontiers of Computer Science \& Technology 7, 630-638 (2013).

48. L. Breiman. Random Forests. Machine Learning 45, 5-32 (2001).

49. S. H. Guo et al. iNuc-PseKNC: a sequence-based predictor for predicting nucleosome positioning in genomes with pseudo k-tuple nucleotide composition. Bioinformatics 30, 1522-1529 (2014).

50. H. Lin, E. Z. Deng, H. Ding, W. Chen \& K. C. Chou. iPro54-PseKNC: a sequence-based predictor for identifying sigma-54 promoters in prokaryote with pseudo k-tuple nucleotide composition. Nucleic Acids Research 42, 12961-12972 (2014).

51. H. Tang, W. Chen \& H. Lin. Identification of immunoglobulins using Chou's pseudo amino acid composition with feature selection technique. Mol Biosyst 12, 1269-75 (2016).

52. P. P. Zhu et al. Predicting the subcellular localization of mycobacterial proteins by incorporating the optimal tripeptides into the general form of pseudo amino acid composition. Molecular Biosystems 11, 558-563 (2015).

53. W. Chen, P. Feng, H. Ding, H. Lin \& K. C. Chou. iRNA-Methyl: Identifying N(6)-methyladenosine sites using pseudo nucleotide composition. Anal Biochem 490, 26-33 (2015).

54. W. Chen, P. Feng \& H. Lin. Prediction of replication origins by calculating DNA structural properties. FEBS Lett 586, 934-8 (2012).

55. W. Chen, P. M. Feng, H. Lin \& K. C. Chou. iSS-PseDNC: identifying splicing sites using pseudo dinucleotide composition. Biomed Res Int 2014, 623149 (2014)

56. Tamanna \& J. Ramana. MATEPRED-A-SVM-Based Prediction Method for Multidrug And Toxin Extrusion (MATE) Proteins. Computational Biology \& Chemistry 58, 199-204 (2015).

\section{Acknowledgements}

The work is supported by National Natural Science Foundation of China (Grant Nos 61472333, 5140540, 71103154 and 41476118) and the Project of Fujian Provincial Department of Science and Technology (Grant No. 2016NZ0001-4) and the Natural Science Foundation of Fujian Province of China (No. 2016J01152).

\section{Author Contributions}

X.R.L. conceived and designed the experiments. Y.Y.C. performed the experiments and wrote the manuscript. Y.J., J.L. and Y.Y.C. analyzed the data and proofread models. Y.M. and Z.J.L. provided more data and experiment. All authors discussed the results, revised and approved the final manuscript. 


\section{Additional Information}

Competing financial interests: The authors declare no competing financial interests.

How to cite this article: Cai, Y. et al. Resistance gene identification from Larimichthys crocea with machine learning techniques. Sci. Rep. 6, 38367; doi: 10.1038/srep38367 (2016).

Publisher's note: Springer Nature remains neutral with regard to jurisdictional claims in published maps and institutional affiliations.

(c) (i) This work is licensed under a Creative Commons Attribution 4.0 International License. The images or other third party material in this article are included in the article's Creative Commons license, unless indicated otherwise in the credit line; if the material is not included under the Creative Commons license, users will need to obtain permission from the license holder to reproduce the material. To view a copy of this license, visit http://creativecommons.org/licenses/by/4.0/

(C) The Author(s) 2016 